Sir,

\section{Preschool vision filtering and amblyopia}

We read with interest your articles by Searle et $a l^{1}$ and Gregson, ${ }^{2}$ with respect to amblyopia treatment and compliance. We appreciate that there is a need to improve compliance and 'self-efficacy' with respect to patching. In Searle et al's study population, the mean visual acuity at first assessment was $6 / 24$, which he had alluded to as being severe, substantial visual loss. Further, preschool children with amblyopia have been shown to be at risk for further deterioration. ${ }^{3}$ For these reasons, we feel it is important to detect amblyopia early.

Recent UK guidelines ${ }^{4}$ have advised that all children be screened before 5 years of age. The French Health Authorities have instituted offering vision screening at 9 months to all infants, as they found that at 9 months, infants were more responsive to the acuity card procedure; further they complied more easily with orthoptic and ophthalmic examination than at a later age. In addition to this, occlusive treatment before 1 year of age has been shown to be more acceptable, with better compliance and a shorter occlusion period, making it more efficient. ${ }^{5}$

Compliance has been stated to be the most critical factor for predicting a successful outcome ${ }^{6}$ with parental nonconcordance and response efficacy having a negative impact. ${ }^{1,7}$ Searle et al argue that the decision to postpone screening until 5 years of age may be premature in the light of poor compliance and not a result of ineffective treatment. Can we achieve better compliance by early recognition and parental education? It is interesting to note that in the same study, $73 \%$ of the children had a family history of amblyopia.

Since 1989, Coventry has an effective preschool vision filtering programme. It allows for opportunistic assessment or self-referral to a community orthoptist. The attendance rate averages $70 \%$. In all, 880 children are screened annually in the programme. Approximately $20 \%$ of children screened are identified as strabismic or anisometropic amblyopes and referred for further assessment to the Paediatric Eye Clinic. Other ocular pathology identified included epiphora $(2.7 \%)$, nystagmus $(1.1 \%)$, and an abnormal red reflex $(0.8 \%){ }^{8}$

We contend that the benefits of preschool filtering should not be overlooked as a valuable aspect of the future provision of children's eye services. While it is important to treat amblyopia effectively, it is also important to detect it early. Besides amblyopia, decreased vision due to refractive errors, strabismus or ocular pathology must be included in any analysis of the cost effectiveness of preschool vision filtering. Where existing effective pre-school programmes exist, these should not be replaced with the year- 5 screening programme; they should run together for the benefit of all young children and not just those of school age.

\section{References}

1 Searle A, Norman P, Harrad R, Vedhara K. Psychosocial and clinical determinants of compliance with occlusion therapy for amblyopic children. Eye 2002; 16: 150-155.

2 Gregson R. Why are we so bad at treating amblyopia. Eye 2002; 16: 461-462.

3 Simons K, Preslan M. Natural history of amblyopia untreated owing to lack of compliance. Br J Ophthalmol 1999; 83: 582587.

4 Children's Eye Health Working Party. Guidelines for Children's Eye Care. Royal College of Ophthalmologists, London. May 2002.

5 Vital-Durand F, Ayzac L. Trackling amblyopia in human infants. Eye 1996; 10: 239-244.

6 Lithander J, Sjorstrand J. Anisometropic and strabismic amblyopia in the age group 2 years and above: a prospective study of the results of treatment. Br J Ophthalmol 1991; 75: 111-116.

7 Newsham D. A randomised controlled trial of written information: the effect on parental non-concordance with occlusion therapy. Br J Ophthalmol 2002; 86: 787-791.

8 Mowatt L, Mac Farlane C, Robinson R. Pre School Vision Filtering, A Necessity in Coventry and elsewhere. Poster presentation at the Annual Congress of Ophthalmologists, Manchester, May 2002.

L Mowatt, C Mac Farlane and R Robinson

Coventry and Warwickshire Hospital, University Hospitals Coventry and Warwickshire NHS Trust Stoney Stanton Road, Coventry CV14FH UK

Correspondence: R Robinson

Tel: + 44 02476224055;

Fax: + 4402476844119

Email: Rosemary.Robinson@uhcw.nhs.uk

Eye (2005) 19, 589. doi:10.1038/sj.eye.6701507

Published online 8 April 2005

Sir,

Reply to preschool vision filtering and amblyopia

We agree with Mowatt et al that compliance with occlusion is often better in younger children. Although vision testing at school entry is easier, coverage is higher, and treatment of amblyopia is still 